\title{
Resiliencia de los municipios costeros del Pacífico mexicano ante desastres socionaturales
}

\section{Resilience to socio-natural disasters of Mexican Pacific coastal municipalities}

\author{
Karen Velázquez-GonZÁLeZ* \\ Salvador Adame-Martínez** \\ Guadalupe Hoyos Castillo** \\ Georges Seingier*
}

\begin{abstract}
This research determined resilience to socio-natural disasters of the coastal municipalities of the Mexican Pacific, through an index built with 24 indicators, congregated in three subindex: prevention, resistance and recovery. The resilience found was mainly medium, due to a weak recovery capacity. A cluster analysis defined five groups with particular resilience characteristics, highlighting one with major lag in Oaxaca and Guerrero due to its low prevention and recovery levels, which will allow decision makers to guide strategies and prioritize actions to increase resilience to natural threats in Mexican territory.
\end{abstract}

Keywords: socio-natural disasters, coastal municipalities, resilience.

\begin{abstract}
Resumen
Este estudio determinó, mediante un índice constituido por 24 indicadores, congregados en tres índices de segundo orden: prevención, resistencia y recuperación, la resiliencia ante desastres socionaturales de municipios costeros del Pacífico mexicano. La resiliencia encontrada fue mayormente media debido a una capacidad de recuperación endeble. Un análisis clúster precisó cinco grupos con características propias de resiliencia, resaltó uno de mayor rezago en Oaxaca y Guerrero debido a su bajo nivel de prevención y recuperación, los hallazgos permiten a quienes toman decisiones orientar estrategias y priorizar acciones para aumentar la resiliencia ante las amenazas naturales en el territorio mexicano.
\end{abstract}

Palabras clave: desastres socionaturales, municipios costeros, resiliencia.

\footnotetext{
*Universidad Autónoma de Baja California, correos-e: karen.velazquez@uabc.edu.mx, georges@ uabc.edu.mx

** Universidad Autónoma del Estado de México, correos-e: sadamem@uaemex.mx, gdhoyos@ uaemex.mx
} 


\section{Introducción}

Los fenómenos naturales a nivel mundial han incrementado su incidencia y magnitud en las últimas décadas; en 2018 se reportaron 315 desastres que dejaron a su paso 11,804 pérdidas humanas, 68 millones 500 mil personas afectadas, pérdidas económicas que ascendieron a 132 billones de dólares, deterioro en infraestructura, interrupción de servicios básicos de seguridad, salud, educación y daños en el tejido social (CRED, 2018). En los últimos años se han registrado avances en la reducción del riesgo de desastres, no obstante, Latinoamérica continúa siendo de las regiones más vulnerables a amenazas naturales, las pérdidas que aquí se registran representan una cuarta parte de las pérdidas globales (FAO, 2017).

Las entidades costeras de México representan 56\% del territorio nacional, concentran $46 \%$ de la población; en ellas se sitúan 158 municipios con acceso directo al mar cuya población asciende a 18 millones 900 mil (Azuz-Adeath et al., 2019); en estas entidades se registran deslizamientos, sismos, ciclones tropicales, inundaciones, lluvias y tormentas severas; los fenómenos hidrometeorológicos son los causantes de $84.4 \%$ de daños y pérdidas registradas en 2018, los ciclones tropicales son los de mayor recurrencia y costo. Si nos referimos a declaratorias emitidas entre 2000 y 2020, los estados con mayor incidencia de desastres son Oaxaca, Chiapas, Sonora y Guerrero en el Pacífico, en conjunto con Veracruz y Yucatán en el Golfo de México (Cenapred, 2020).

Por su parte, la gestión del riesgo de desastres en México transita de una perspectiva reactiva a un enfoque de prevención dando cabida a la resiliencia, término utilizado ampliamente desde diversos ámbitos, que se remonta a estudios sobre sistemas ecológicos y refiere una propiedad positiva que permite tanto el desarrollo como la conservación de un sistema (Metzger y Robert, 2013). En la década de los 90, fue asociada a situaciones que ponen en riesgo a los seres humanos (Contreras y Beltrán, 2015), debe ser vista como la capacidad del sistema para manejar los impactos antes de la crisis, lo que permite reducir significativamente los costos y consecuencias de los desastres y no centrarse en la respuesta a la emergencia (Clark-Ginsberg et al., 2020).

De igual manera, las investigaciones sobre resiliencia han adoptado diversos enfoques: comunitario, social, económico, urbano, socio-ecológico, cambio climático, estructural, ingeniería civil o como componente de la vulnerabilidad (Chen et al., 2020; Cutter et al., 2010; Lu et al., 2020; Maldonado y González, 2013; Santiago Vera et al., 2018). En consecuencia, para medir la resiliencia, han surgido marcos e instrumentos enfocados en diferentes fenómenos perturbadores y a múltiples escalas, desde modelos basados en indicadores, evaluación participativa, consultas a expertos, 
evidenciando una creciente área de investigación (Clark-Ginsberg et al., 2020; Copeland et al., 2020).

La conceptualización de la resiliencia marca las diferencias en la manera de abordarse, sin embargo, pudiera agruparse en tres categorías como proponen Patel et al. (2017) y Uriarte (2013): 1) Resiliencia como estabilidad, entendida como la capacidad de asimilar o absorber daños de determinada magnitud. 2) Resiliencia como recuperación, referida a la capacidad de regresar al estado original; y 3) Resiliencia como transformación, implica la capacidad de resistir a las amenazas y fortalecerse de la situación.

Existe una definición integradora que considera las tres categorías anteriores, donde la resiliencia es concebida como la capacidad del sistema para prevenir, absorber/resistir los impactos, recuperarse rápidamente (Chen et al., 2020 y Labaka et al., 2015) y aprender de los efectos de los peligros (Jones et al., 2013), sin pretender volver al estado inicial o lograr una estabilidad estática, sino lograr un estado mejor, visión desde la cual se aborda esta investigación.

Estas etapas representan el ciclo de vida de la resiliencia (Labaka et al., 2015) que, acorde con González y Franco (2017), en el tiempo conciernen 1) al antes del desastre, momento centrado en la preparación y reducción desde un enfoque preventivo, la inversión en esta etapa disminuye los costos de los desastres de tres a ocho veces; 2 ) durante el desastre, donde es necesaria una respuesta efectiva con enfoque proactivo y correctivo y 3) post desastre, correspondiente a la recuperación y aplicación de soluciones correctivas y preventivas, vislumbrando la posibilidad de que se presente un nuevo evento.

México, al igual que diversos países latinoamericanos, se encuentra alineado con los esfuerzos mundiales sobre reducción del riesgo de desastre entre los que destacan el Plan de Acción de Yokohama para un Mundo más Seguro de la Primera Conferencia Mundial sobre Reducción del Riesgo, celebrada en Yokohama Japón, en 1994; el Marco de Acción de Hyogo (MAH) en 2005, derivado de la Segunda Conferencia Mundial sobre Reducción del Riesgo en Kobe, Japón; más tarde, en 2015, el Marco de Acción de Sendai (MAS), como parte de la Tercera Conferencia Mundial sobre Reducción del Riesgo en Sendai, Japón.

Los avances en la implementación de estos instrumentos internacionales evidencian el desarrollo de acciones: impulso de la preparación ciudadana mediante brigadas comunitarias, estudios de reubicación de población en riesgo, mejoramiento de infraestructura, recopilación de información local sobre riesgo y resiliencia mediante la aplicación de encuestas a pobladores como evidencia de la implementación del Marco de Sendai y otras agendas globales, así como la creación de fondos de reconstrucción (cuadro 1). 


\section{Cuadro 1}

\section{Acciones desarrolladas para reducir el riesgo de desastre y aumentar la resiliencia en México}

\begin{tabular}{cl}
\hline Año & \multicolumn{1}{c}{ Acción } \\
\hline 2007 & $\begin{array}{l}\text { Taller de gestión de riesgos de } \\
\text { desastres }\end{array}$ \\
& \\
& Red Nacional de Brigadistas \\
& Comunitarios de Protección \\
& Civil \\
& \\
& Programa Municipio Seguro: \\
& Resistencia a Desastres \\
& \\
& Fideicomiso del Fondo de \\
& Reconstrucción de Entidades \\
& Federativas (FREF) \\
& $\begin{array}{l}\text { Programa de Prevención de } \\
\text { Riesgos en los Asentamientos } \\
\end{array}$ \\
& Humanos (PRAH)
\end{tabular}

2013 Estrategia: México seguro frente a desastres

2014 Programa de Reubicación de la Población en Zonas de Riesgo (Repzor)

2016 Taller Implementando el marco de Sendai a nivel local: formulación de planes de acción para la resiliencia

Guía de Resiliencia Urbana

Mejorar la preparación de la ciudadanía en la prevención; se promueven espacios para desarrollar acciones básicas de prevención y anticipación del riesgo.

Impulsar la inclusión de la gestión integral del riesgo de desastres en prácticas y políticas de la Administración Pública Federal (APF).

Reducir el riesgo de desastres en las localidades y municipios, vinculando esfuerzos de la sociedad, gobierno local e iniciativa privada.

Ofrecer crédito a las entidades federativas afectadas por desastres a un plazo de 20 años, del cual únicamente pagarán los intereses.

Fomentar las capacidades municipales en la prevención del riesgo mediante obras que reduzcan la vulnerabilidad de la población y aumenten la resiliencia.

Contribuir a mejorar la resiliencia de la infraestructura y servicios públicos ante fenómenos naturales.

Instaurar directrices para incidir a nivel estatal en la elaboración de Programas de Ordenamiento Territorial y estudios de factibilidad para la reubicación de la población en zonas de riesgo. En 2015 se transformó en el Programa de Ordenamiento Territorial y Esquemas de Reubicación de la Población en Zonas de Riesgo (POTER).

Cumplir con la meta de 122 Planes de Acciones Municipales de Resiliencia, así como dar a conocer la campaña mundial "Desarrollando ciudades resilientes".

Servir como marco de actuación para los municipios, a fin de diseñar e implementar acciones para prevenir el riesgo, minimizar su impacto en la población, así como establecer mejores esquemas de recuperación. 
Cuadro 1 (continuación)

\begin{tabular}{|c|c|c|}
\hline Año & Acción & Propósito \\
\hline 2016 & $\begin{array}{l}\text { Programa de Prevención de } \\
\text { Riesgos }\end{array}$ & $\begin{array}{l}\text { Fomentar acciones de planeación, preven- } \\
\text { ción y ocupación adecuada del territorio, y } \\
\text { así disminuir la vulnerabilidad de los asen- } \\
\text { tamientos humanos. Surge de la fusión del } \\
\text { PRAH y POTER. }\end{array}$ \\
\hline 2017 & $\begin{array}{l}\text { Guía para la elaboración del } \\
\text { Plan de Acción Local de Resi- } \\
\text { liencia }\end{array}$ & $\begin{array}{l}\text { Facilitar la elaboración de los planes de } \\
\text { acciones municipales de resiliencia. }\end{array}$ \\
\hline 2019 & $\begin{array}{l}\text { Programa Visión de Primera } \\
\text { Línea }\end{array}$ & $\begin{array}{l}\text { Monitorear el progreso de la resiliencia } \\
\text { comunitaria, a partir del establecimiento de } \\
\text { una línea base local, desde un enfoque } \\
\text { inclusivo centrado en las personas. }\end{array}$ \\
\hline
\end{tabular}

Fuente: elaboración propia con datos de DOF, 2015; GNDR, 2015; Gobierno Federal, 2013; 2008.

No obstante la institucionalidad, en México, a nivel local, la implementación del MAH y MAS ha sido incipiente quedando así varios puntos por reforzar, es así que, en 2012, se redujo 75\% el presupuesto del Programa de Prevención de Riesgos en los Asentamientos Humanos, solo 15\% de los 2457 municipios existentes contaban al 2019 con Atlas de Riesgo Municipal, mientras 89 municipalidades estaban incorporadas al Programa Municipio Seguro: Resistente a Desastres, una de las medidas principales para promover la gestión integral del riesgo (Medina et al., 2017; OCDE, 2013).

Para transitar de una visión reactiva a una preventiva, México es de los pocos países latinoamericanos que cuenta con herramientas presupuestarias tanto de recuperación (Fonden) como prevención (Fopreden) (Fundación idea, 2017), no obstante, se continúa gastando más en la respuesta al desastre y la necesidad de invertir en prevención continúa desatendida. Durante el periodo 2008-2018, el presupuesto del Fopreden representó 5\% del presupuesto del Fonden, esto puede relacionarse con un problema político: el peso político a futuro de la atención post desastre y la reconstrucción es más grande que el de la prevención.

En relación con lo anterior, un elemento fundamental para llevar a cabo cualquier política, que emane de acuerdos internacionales no vinculantes o de iniciativas locales, es la solidez institucional (entre niveles de gobierno, actores y organismos de la sociedad civil) (Baas et al., 2009), la cual es necesaria para lograr una gestión del riesgo adecuada: la transición de la gobernabilidad hacia la gobernanza, al integrar en el diálogo a quienes viven los efectos de los impactos, permitirá construir mecanismos adaptados a cada región que reflejen la diversidad latinoamericana. 
El Marco de Sendai introduce el reconocimiento de que los arreglos institucionales deficientes se transforman en factores subyacentes del riesgo; igualmente, refuerza el rol de la toma de decisión informada y sensible al riesgo para la evaluación ex ante de inversiones tanto públicas como privadas. En esta línea, es interesante mencionar que el Marco de Sendai reconoce la naturaleza política del manejo del riesgo, por lo que se requiere una mayor transparencia, una definición clara de responsabilidades entre sectores y una participación de los diferentes actores para, de esta forma, asegurar la rendición de cuentas ante la generación de nuevos riesgos, asimismo, existe una necesidad por integrar la reducción del riesgo a desastres dentro del proceso de planificación del desarrollo y en los esfuerzos sectoriales que lo comprende (FAO, 2017).

El presente estudio tiene como objetivo realizar un diagnóstico del grado de resiliencia ante desastres socionaturales que presentan los municipios costeros de la región del Pacífico mexicano, a través del desarrollo y aplicación de un índice basado en indicadores agrupados, a su vez, en tres índices de segundo orden que evidencian la capacidad de prevención, resistencia y recuperación, se toman como punto de partida las directrices establecidas en metodologías desarrolladas a nivel internacional y nacional. De igual manera, se busca definir el nivel de atención que requieren los 88 ayuntamientos costeros en función de la capacidad existente para orientar estrategias y priorizar acciones en la construcción de municipios resilientes.

\section{Metodología}

La zona Pacífico es una de las tres grandes regiones del país, la línea de costa del océano Pacífico transcurre por 11 entidades federativas donde 88 municipios son costeros, es decir, interactúan con las dinámicas del mar. La temporada de ciclones tropicales en el océano Pacífico comprende de mayo a noviembre, no obstante, las temperaturas registradas en la superficie del mar en la región sur hacen posible la presencia de ciclones tropicales todo el año. En función del número de tormentas tropicales y huracanes ocurridos en el periodo de 1949 a 2000, el océano Pacífico registró un mayor número respecto al Atlántico en una proporción de seis veces (Rosengaus et al., 2014).

En el caso de los fenómenos geológicos, de acuerdo con la regionalización sísmica de México, la región del Pacífico corresponde a las zonas B y $\mathrm{C}$ catalogadas como intermedias, donde los sismos registrados no resultan tan frecuentes, y D, donde históricamente se han reportado grandes sismos, los cuales además ocurren de manera frecuente (SGM, 2017). 
La evaluación de resiliencia se efectuó a nivel municipal, dadas las atribuciones del municipio para establecer medidas preventivas que permitan hacer frente a los desastres socionaturales, fomentar la participación de la ciudadanía, constituir canales efectivos de comunicación, incluir la Gestión Integral del Riesgo de Desastres en la planeación del territorio, tutelar el cambio de uso de suelo, aplicar y verificar el cumplimiento de los diferentes reglamentos como el de construcción, fundamental para crear sitios resilientes.

\subsection{Selección de indicadores}

El índice de evaluación de resiliencia se cimentó en indicadores, los cuales tienen como objeto simplificar y comunicar la realidad de una situación compleja, mostrar la posición relativa del fenómeno a medir e ilustrar la magnitud o dirección de cambio (Cutter et al., 2010). Originalmente se recopilaron, de una revisión de metodologías desarrolladas a nivel internacional y nacional enfocadas en la resiliencia, 129 indicadores, de los cuales se seleccionaron 24 en función de tres criterios: relevancia en el fortalecimiento de la capacidad de resiliencia manifestada en la literatura, confiabilidad de datos y disponibilidad de información, ya que como puntualizan Schuschny y Soto (2009), la fortaleza de un índice recae en la calidad de las variables que lo definen, por lo que su selección debe fundamentarse en su relevancia, calidad, frecuencia de muestreo y su disponibilidad al dominio público. De acuerdo con su naturaleza, los indicadores se clasificaron en institucionales, legales, sociales, económicos, estructurales, integradores, ambientales y de infraestructura.

A juicio de los autores Labaka et al. (2015) y Twigg (2007), la planeación, a la par del sistema legal y regulatorio, debe ser un componente a considerar en la evaluación de la resiliencia como parte de la gobernabilidad. En este tenor, se propusieron cinco indicadores en el componente de prevención, que en conjunto inciden en la no construcción del riesgo; reflejan la presencia, o ausencia, de la planeación integral del riesgo en la agenda municipal, así como la consciencia de que el riesgo cambia con el tiempo y, por tanto, se requiere la actualización de los instrumentos: 1) El plan municipal de desarrollo, que permite un diagnóstico de las principales problemáticas en materia de riesgo, el plan ideal contempla entre sus ejes la protección civil; 2) El atlas de riesgo resulta relevante en el diagnóstico de amenazas, vulnerabilidad e identificación de zonas de riesgos; 3) El reglamento de protección civil regula acciones de prevención y salvaguarda de personas, bienes materiales y medio ambiente; 4) La regulación del uso de suelo es relevante dado que previene la proliferación de asentamientos en zonas de riesgo y el deterioro de ecosistemas, al 
establecer una zonificación; 5) El reglamento de construcción, se consideró ya que proporciona la normativa de seguridad mínima, la cual contempla zonas de riesgo no aptas para la construcción.

La información con que se trabajó provino de bases de datos de acceso público con características disímiles, expresadas en unidades de medidas diferentes, esto es, dicotómicas (presencia-ausencia), económicas, porcentajes, longitud, entre otras, las cuales no podían congregarse de esta manera. La estandarización de datos se ejecutó mediante el método denominado categorización de escalas (Schuschny y Soto, 2009), este método asigna a cada indicador una categoría y un valor en función de su cercanía a la condición óptima deseable, en este caso se trabajaron tres categorías: bajo, medio y alto, con valores de $0,0.5$ o 1 , respectivamente, a excepción de los indicadores dicotómicos, en que se evaluó la ausencia (0) o presencia (1) (cuadro 2).

La consistencia interna del índice general de resiliencia se evaluó mediante el coeficiente Alfa de Cronbach, éste indica qué tan bien está representada la información de las variables elegidas en un solo índice; toma valores de 0 a 1 , donde valores cercanos a uno indican mayor fiabilidad en la selección de indicadores, por tanto, las variables propuestas miden correctamente el fenómeno de interés (Schuschny y Soto, 2009). El coeficiente obtenido fue de 0.79 , por arriba del valor mínimo aceptable (0.70).

\subsection{Componentes de la resiliencia y método de agregación}

Para evaluar la situación de resiliencia se consideraron tres índices de segundo orden: Índice de Prevención (IPre), Índice de Resistencia (IRes) e Îndice de Recuperación (IRec), correspondientes a las etapas del ciclo de resiliencia establecidos por Labaka et al., (2015).

El índice de prevención refiere el conjunto de medidas o acciones desarrolladas e implementadas con anticipación que evitan o disminuyen los efectos negativos que pudiera originar un desastre socionatural sobre la población, ambiente, bienes y servicios; éste consideró nueve indicadores de índole legal e institucional sobre desarrollo, planeación y construcción de infraestructura en el municipio. Entre las acciones estimadas dentro del indicador de cultura de prevención, referente a actividades implementadas por el gobierno municipal, se encuentran la ejecución de simulacros, impartición de cursos de prevención, coordinación de programas educativos preventivos, concientización sobre el uso de suelo, mesas sectoriales de información y pláticas de concientización, asimismo, acciones para impulsar la autoprotección y protección corporativa. La difusión de la información, por su parte, se evaluó en función de tres medios de difusión: impresos, masivos (radio y televisión) y aviso casa por casa. 


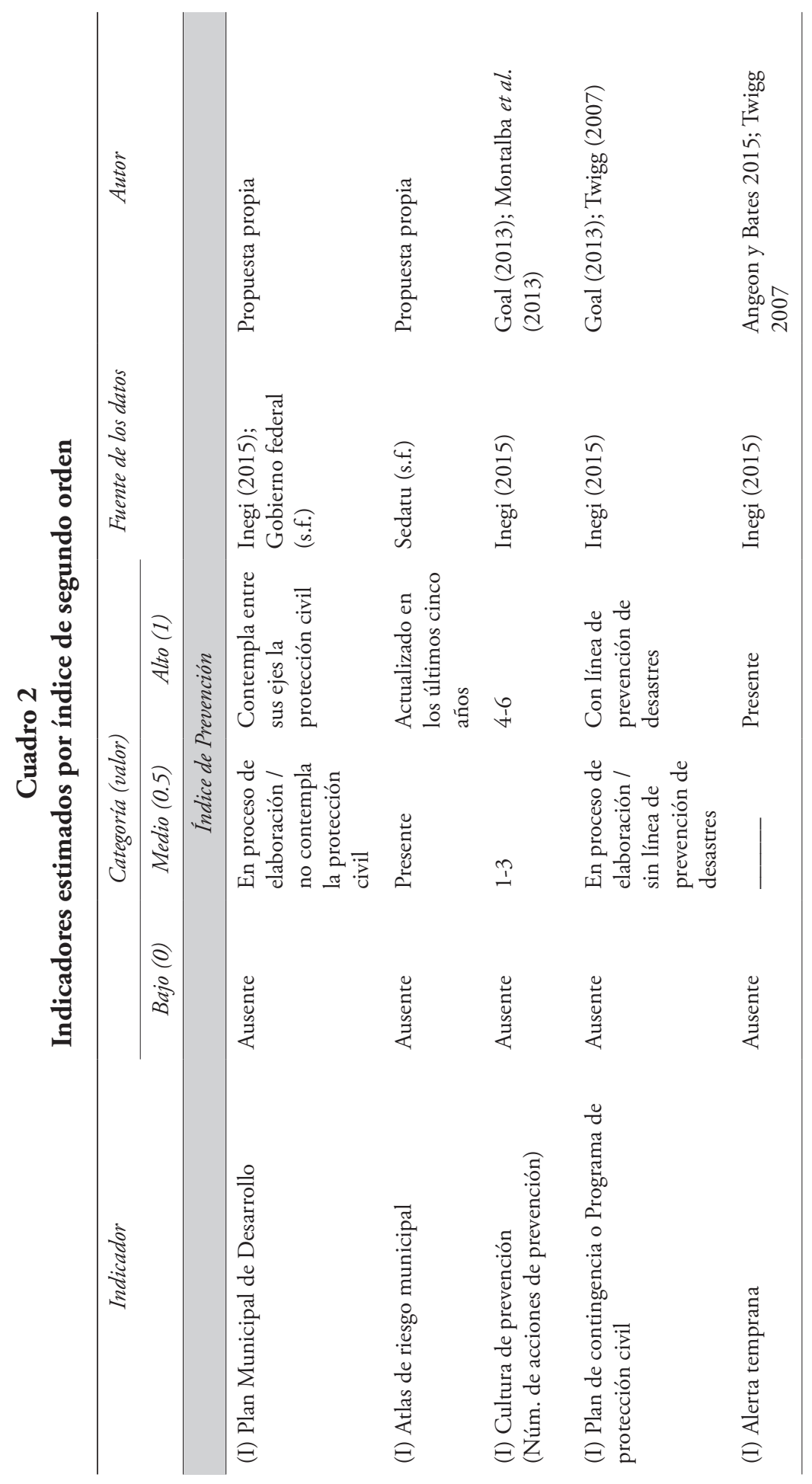




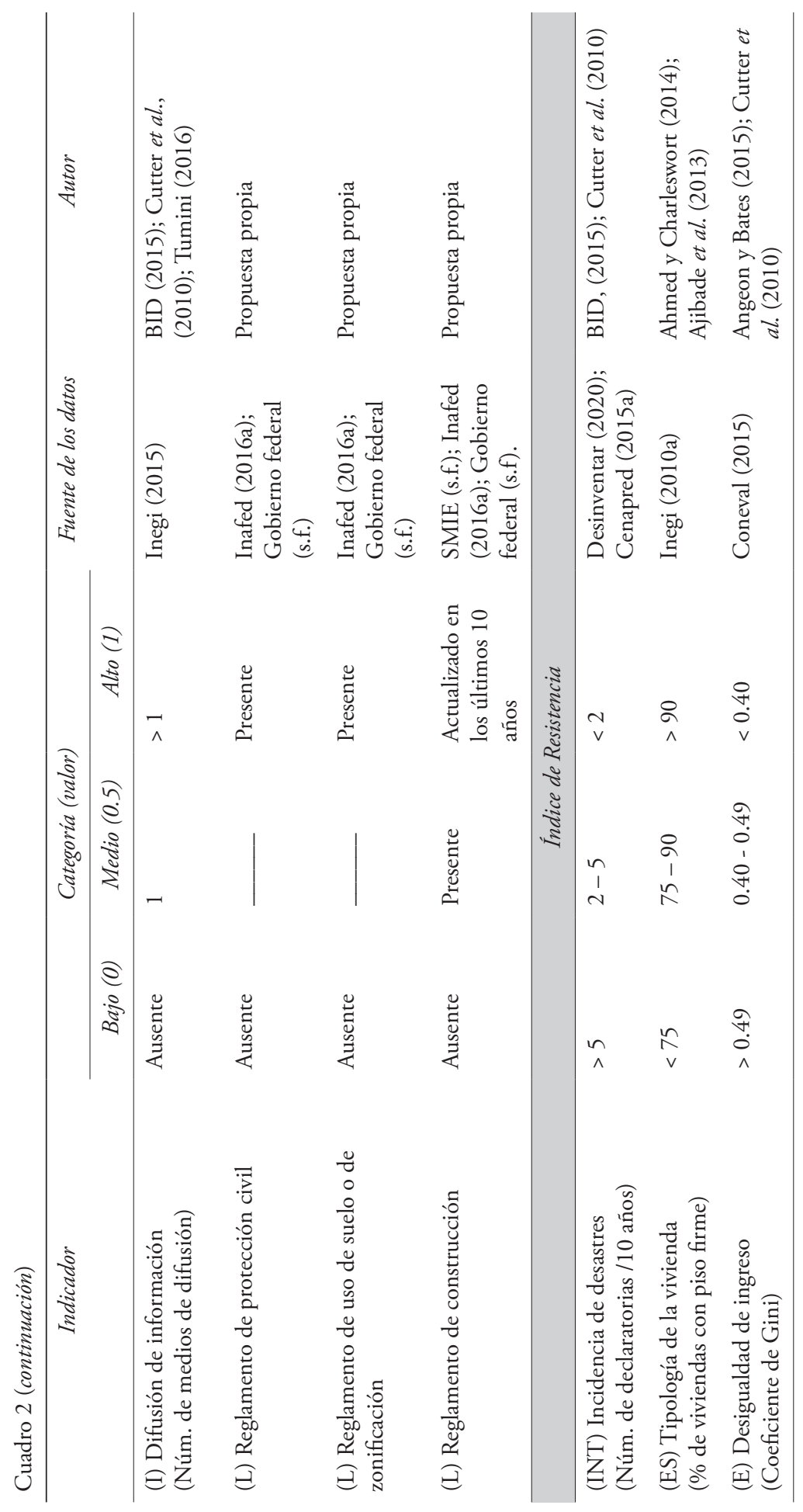




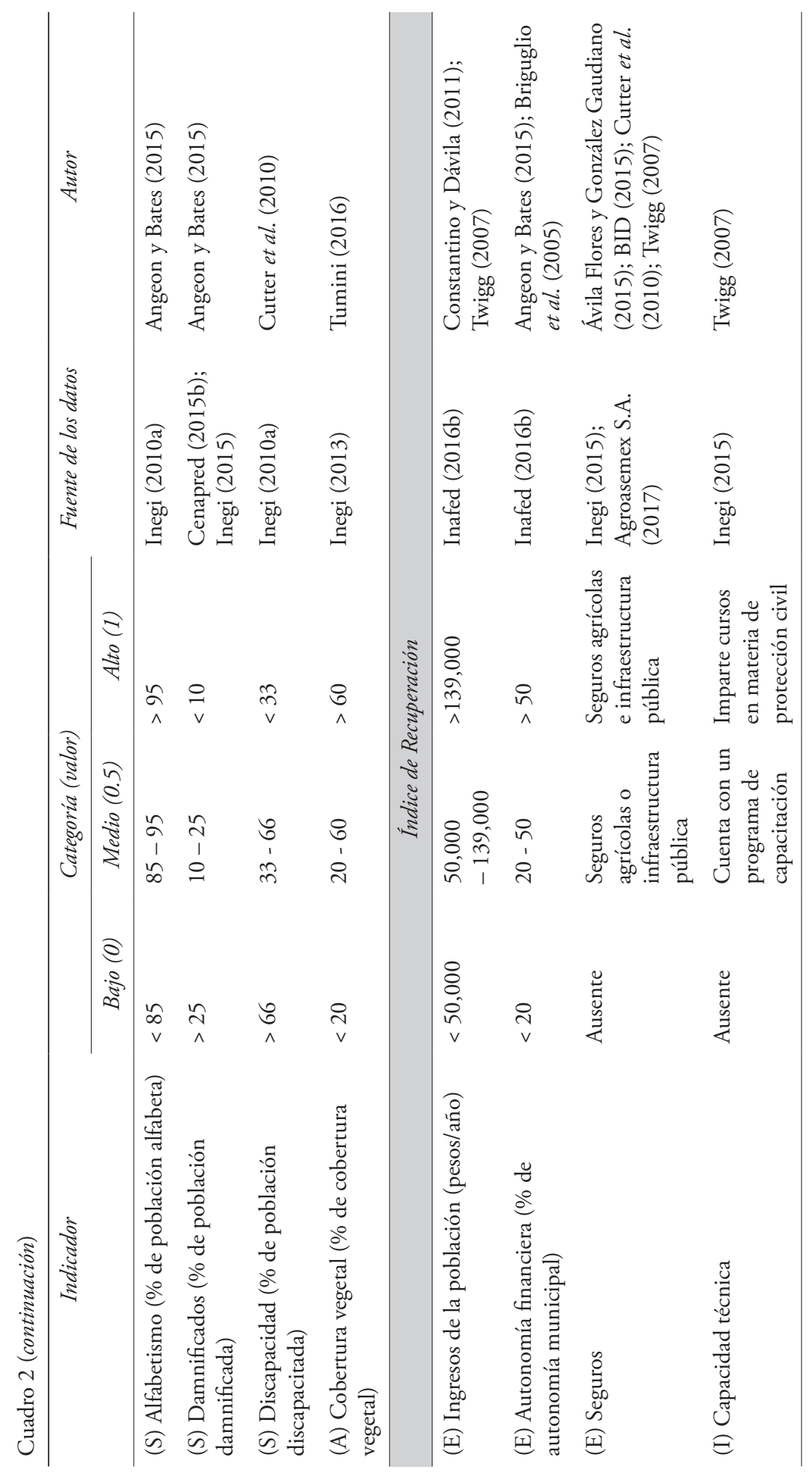




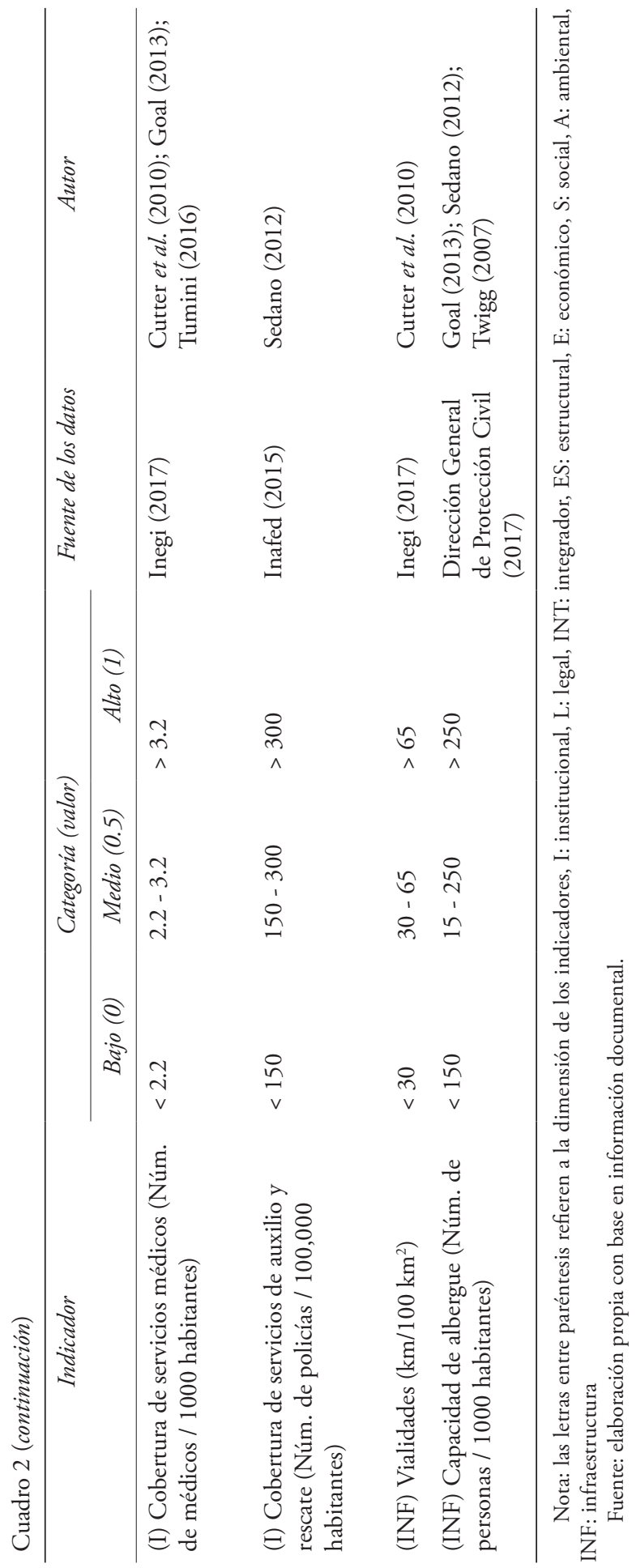


El índice de resistencia contempló siete indicadores correspondientes a las dimensiones social, económica, estructural, ambiental y un indicador integrador. Este índice mostró la capacidad del sistema socionatural para reaccionar de manera adecuada, con eficacia y rapidez, ante la presencia de un fenómeno perturbador y así persistir ante sus efectos. Sugiere que municipalidades con mejores condiciones ambientales, combinadas con altos niveles de igualdad económica, educativa de la población y un menor número de habitantes con limitaciones para realizar tareas de la vida cotidiana, son más resilientes.

El índice de recuperación consideró las acciones o mecanismos de rehabilitación desplegadas inmediatamente después de concluida la fase de emergencia, con el objeto de lograr el mejoramiento y restauración de infraestructura, medios de sustento y condiciones de vida de la población afectada; se construyó a partir de ocho indicadores institucionales, infraestructurales y económicos. Resulta conveniente subrayar que los niveles de recuperación y resistencia pueden variar en función de la intensidad del desastre.

En lo referente a la ponderación de los índices de segundo orden se optó por emplear una ponderación equiproporcional, en la que los tres índices tuvieron el mismo factor de peso (1) en la obtención del índice de resiliencia final, ilustrando así la misma importancia relativa de las tres etapas de la gestión del riesgo de desastre en los municipios, dado que las tres etapas están concatenadas y tienen influencia entre sí. Es cierto que la prevención juega un papel relevante al determinar el grado de impacto que tendrá un desastre en el municipio, no obstante, la manera en que se soportan los efectos del embate influye en la capacidad de recuperación, mientras la etapa de recuperación permite identificar las limitantes en las fases anteriores y reorientar las actividades preventivas futuras.

El método de agregación de la información a nivel de índice de segundo orden correspondió a una media aritmética. Por otro lado, el índice general de resiliencia se obtuvo de la sumatoria de los índices de prevención, resistencia y recuperación, los valores finales se encuentran en un rango de 0 (menos resiliente) a 3 (más resiliente). Las categorías del Índice de Resiliencia se establecieron mediante intervalos equivalentes, al igual que con los indicadores, se definieron tres categorías: alto, medio y bajo, asociadas a una escala cromática: verde, naranja y rojo, correspondientemente, en donde la condición óptima concierne al color verde, o bien, a una resiliencia alta ante desastres socionaturales. 


\subsection{Contribución de los componentes de la resiliencia y similitud entre municipios}

Una vez calculados los diferentes índices (IPrev, IRes e IRec), se determinó la contribución de cada uno al valor final del Índice de Resiliencia, posteriormente se llevó a cabo un análisis jerárquico aglomerativo (análisis clúster) con el paquete estadístico Statistical Package for the Social Sciences (SPSS) (IBM, 2009), con el propósito de determinar la similitud entre los municipios y establecer grupos diferentes entre sí, pero internamente homogéneos, en función de una matriz de asociación de los índices de segundo orden, lo que permite establecer estrategias que respondan a las debilidades identificadas, en prevención, resistencia o recuperación, así como identificar los municipios que requieren atención prioritaria. Los parámetros empleados fueron una distancia euclídea al cuadrado y un método de conglomeración denominado vinculación promedio entre grupos (average linkage), el cual corresponde a la distancia media entre los componentes de cada grupo, con varianzas similares o pequeñas.

\section{Resultados: a medio camino de la resiliencia}

El Índice General de Resiliencia, resultado de la sumatoria de la capacidad de prevención, resistencia y recuperación, manifestó que el nivel medio prevaleció en $82 \%$ (72 municipios) de los municipios costeros, por su parte el nivel bajo quedó representado en 12\% (11 municipios), 6\% restante (cinco municipios) correspondió a resiliencia alta. Sin embargo, aun estos ayuntamientos continúan registrando afectaciones por la presencia de desastres socionaturales, lo cual podría explicarse debido a que, si bien el componente que determina en gran medida su nivel de resiliencia fue la prevención, ésta contempla instrumentos que en ocasiones no son implementados de manera correcta, por lo que habría que darles seguimiento para definir si han logrado su cometido y evaluar realmente su aplicación. La valoración alta se obtuvo debido a valores entre 2.001 y 2.168, cercanos al límite superior de la clase media; el nivel teórico máximo de resiliencia (3) no se alcanzó.

En cuanto a las regiones, la zona Norte no presentó valores bajos de resiliencia, mientras que en el Sur los valores altos estuvieron ausentes. En entidades como Baja California, Colima y Chiapas, la totalidad equivale a nivel medio; en contraparte, la resiliencia baja se localiza en las municipalidades de Michoacán, Guerrero y Oaxaca (mapa 1).

Los resultados obtenidos con el Índice de Prevención muestran que cerca de 55\% de los municipios presenta un nivel medio, localizados en 


\section{Mapa 1}

Índice municipal de resiliencia de la región Pacífico de México (IR). Índice de prevención (IPre), índice de resistencia (IRes), índice de recuperación (IRec)

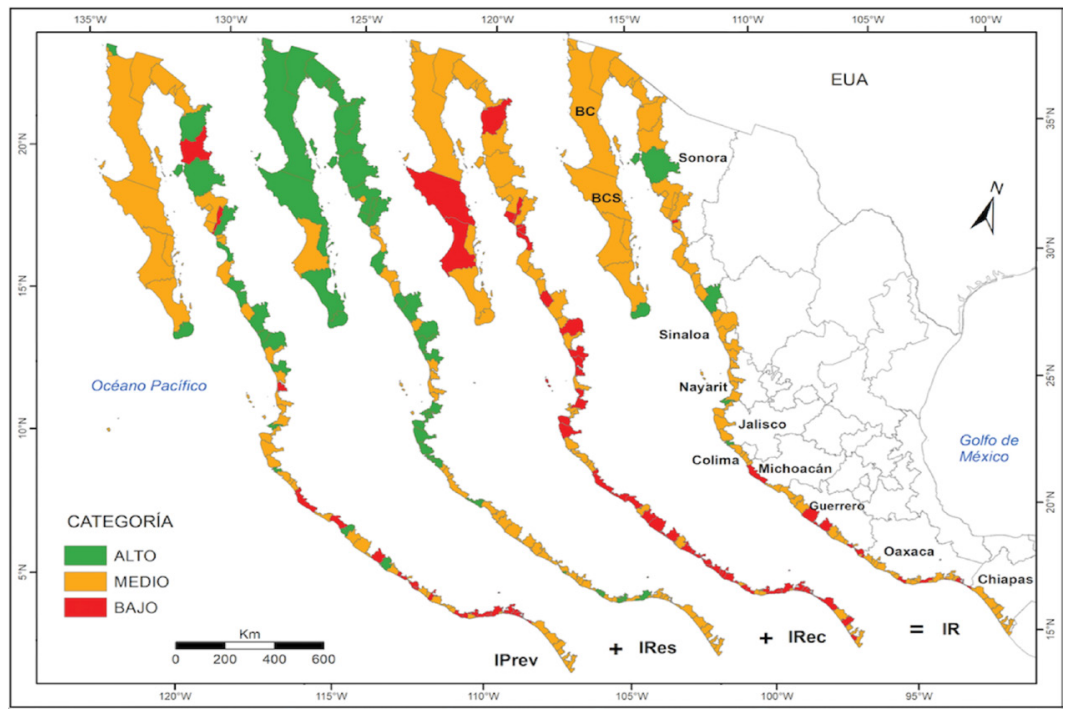

Fuente: elaboración propia con base en el Índice de Resiliencia e Inegi, 2010b.

su mayoría en las regiones Pacífico Sur y Pacífico Norte; en lo referente al nivel bajo y alto concentraron $27 \%$ y $18 \%$, respectivamente, particularmente el nivel bajo se ubicó casi en su totalidad en la región Pacífico Sur, mientras que la distribución geográfica de municipios con capacidad de prevención alta se localiza primordialmente en la región Norte.

En el caso del Índice de Resistencia, ninguno de los municipios costeros estudiados se encontró en nivel bajo, cerca de la mitad se centró en un nivel medio (56\%), alrededor del 38\% ubicado en el Pacífico Sur. Por el contrario, en lo referente al nivel alto la región Norte concentró el 26\%, de esta manera se puede decir que en el norte los valores que predominaron fueron altos, no así en el sur, cuyos valores fueron primordialmente medios, mientras que en el centro se encontraron distribuidos más o menos de manera equitativa entre el nivel medio y alto.

El Índice de Recuperación, a diferencia del índice de resistencia que no registró valores bajos, no mostró en ninguno de los casos niveles altos, es así que predominó una capacidad de recuperación baja (56\%). El nivel bajo se focalizó en la región Sur, el medio en la porción Norte, en el Pacífico Centro las proporciones entre ambos niveles fueron semejantes (cuadro 3). 


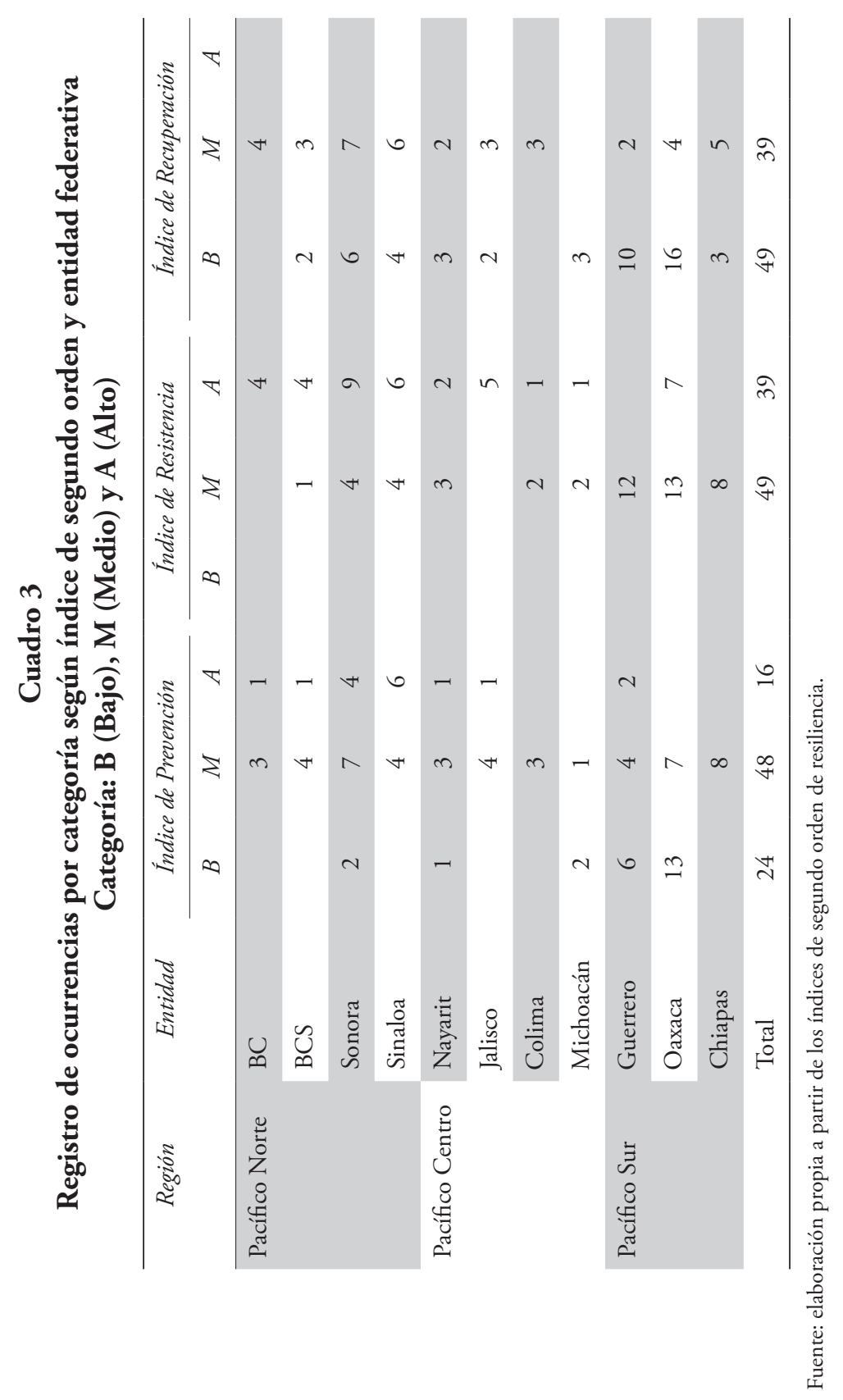


La capacidad de resistencia y prevención contribuyeron de manera equilibrada al índice general de resiliencia en la gran mayoría de los casos analizados, aunque con un sesgo hacia la resistencia (figura 1), por lo que la capacidad de resiliencia estuvo explicada principalmente por el índice de resistencia, primordialmente relacionado con indicadores de índole social, ambiental, económico y estructural, y por el índice de prevención con indicadores legales e institucionales, no así por la recuperación enfocada en la parte institucional, infraestructural y económica.

Figura 1

Contribución de los índices de prevención (IPre), resistencia (IRes) y recuperación (IRec), al índice de resiliencia (IR)

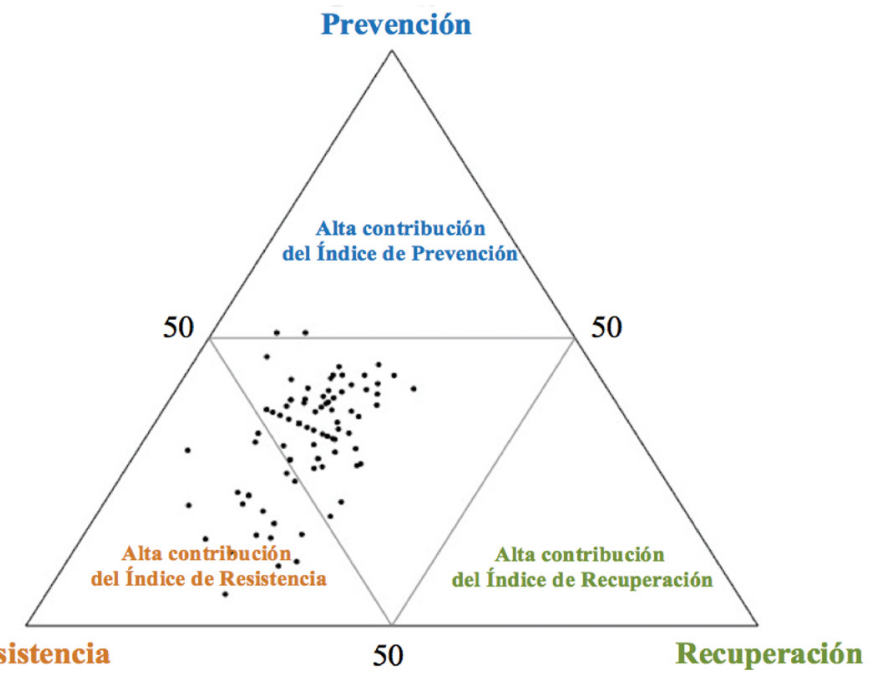

Fuente: elaboración propia a partir de los índices de segundo orden de resiliencia.

De los 24 indicadores seleccionados, cerca de 37\% fueron institucionales y $17 \%$ económicos, en la misma proporción se encuentran legales y sociales (13\%), $8 \%$ infraestructurales y, finalmente, $4 \%$ ambientales, estructurales e integradores, lo que representa más de una tercera parte de indicadores institucionales, puesto que este aspecto juega un papel relevante en la reducción del riesgo de desastres al facilitar una adecuada implementación, o bien, fungir como cuello de botella.

En el análisis a nivel indicador para el índice de prevención, el reglamento de uso de suelo o zonificación resultó ser el más débil, únicamente 17 municipios (19\%), ubicados de manera homogénea en la región Norte y Sur, cuentan con este instrumento de regulación. La difusión del riesgo, seguido del reglamento de construcción, igualmente presentaron valores 
bajos, en el primer caso ocho municipios (9\%) emplean diversos mecanismos para dar aviso a la población: medios masivos impresos y casa por casa; en el segundo, cerca de una sexta parte de los municipios (11 en la Región Norte) tienen reglamento de construcción actualizado, al menos en los últimos 10 ańos. En el extremo opuesto se ubicaron el Plan de Desarrollo, Reglamento de Protección Civil, Plan de Contingencias y Alerta Temprana, todos ellos presentes en más de la mitad de los municipios.

En lo que respecta a los indicadores de resistencia: la discapacidad, damnificados y tipología de la vivienda fueron los mejor evaluados; en todos los casos menos de $9 \%$ de la población presenta limitaciones para las actividades de la vida diaria; en lo concerniente a la población damnificada está por debajo de $10 \%$ en cerca de tres cuartas partes de las municipalidades; las viviendas cuentan con piso firme en más del $70 \%$. El indicador de incidencia de desastres corresponde al más débil, por arriba de la mitad de los municipios registran más de cinco declaratorias de emergencia en promedio, en un periodo de 10 años.

En tanto, en los indicadores de recuperación, la autonomía financiera, vialidades, servicios médicos, así como la capacidad de albergue, se ubicaron en niveles bajos en gran proporción. Lo que indica que $68 \%$ de los ayuntamientos no cuentan con ingresos suficientes para cubrir sus gastos, por ende, no poseen recursos para casos de emergencia.

La densidad de carretera es baja en $72 \%$ de los municipios, por lo que se tienen pocas vías de entrada y salida que sirvan para trasladar a los afectados, víveres o materiales para la reconstrucción. En el mismo tenor, $67 \%$ no cuenta con el número adecuado de médicos que brinden atención necesaria a la población en caso de resultar heridos o afectados, mientras que $84 \%$ no cuenta con espacios suficientes para albergar a la población damnificada, que corresponde al valor de afectados que se tienen en promedio debido a desastres. Los ingresos de la población se ubicaron entre bajo y medio casi de manera equitativa, en caso de desastre los recursos de la población serán insuficientes para comenzar la reconstrucción de sus viviendas con recursos propios y habría que esperar a recibir recursos federales. La capacidad técnica fue el único indicador ubicado en nivel alto, esto denota la impartición de cursos en materia de protección civil.

La construcción de la resiliencia de los municipios costeros del Pacífico mexicano se encuentra a medio camino; aún quedan aspectos por atender como es la implementación efectiva e ininterrumpida de instrumentos y acciones preventivas; se requiere también identificar las acciones que han funcionado adecuadamente durante la etapa de recuperación y aquellas que deben ser reorientadas, principalmente en esta etapa que resultó ser la más débil del ciclo de resiliencia de acuerdo con la evaluación realizada. 


\subsection{Clasificación de los municipios según características de resiliencia}

La evaluación de similitud de las características mostradas por cada municipio costero respecto a la capacidad de prevención, resistencia y recuperación permitió definir cinco grupos con características propias de resiliencia, los cuales fueron priorizados con el propósito de identificar el nivel y tipo de atención requerido. Los clúster 1 y 2 representaron los grupos de mayor prioridad, ambos exhibieron resistencia media y recuperación baja, mientras la prevención -en el primero de los casos- se definió como baja, y nivel medio en el segundo; estuvieron constituidos por 20 y 16 municipalidades, respectivamente, pertenecientes primordialmente a los estados de Oaxaca, Guerrero y Chiapas.

El clúster 3 de prioridad media resultó ser el grupo más pequeño, con dos municipios muy distantes geográficamente hablando, uno de ellos ubicado en Sonora, porción Norte y el segundo en Guerrero, región Sur. Las mejores condiciones de resiliencia las exhibieron los clúster 4 y 5 , congregan 42 y 8 ayuntamientos, correspondientemente, entre ellos los cinco de resiliencia alta. El clúster 5 presenta las mejores condiciones de resiliencia, la capacidad de prevención es la de mayor aporte. Esta sería la situación a promover para dar mayor peso al enfoque preventivo ya que continúan registrándose diversos desastres que dejan a su paso cuantiosas pérdidas, sin dejar de lado el necesario aumento de la resiliencia general, aun en este grupo (mapa 2 y cuadro 4).

\section{Mapa 2}

Nivel de atención requerido según características de resiliencia

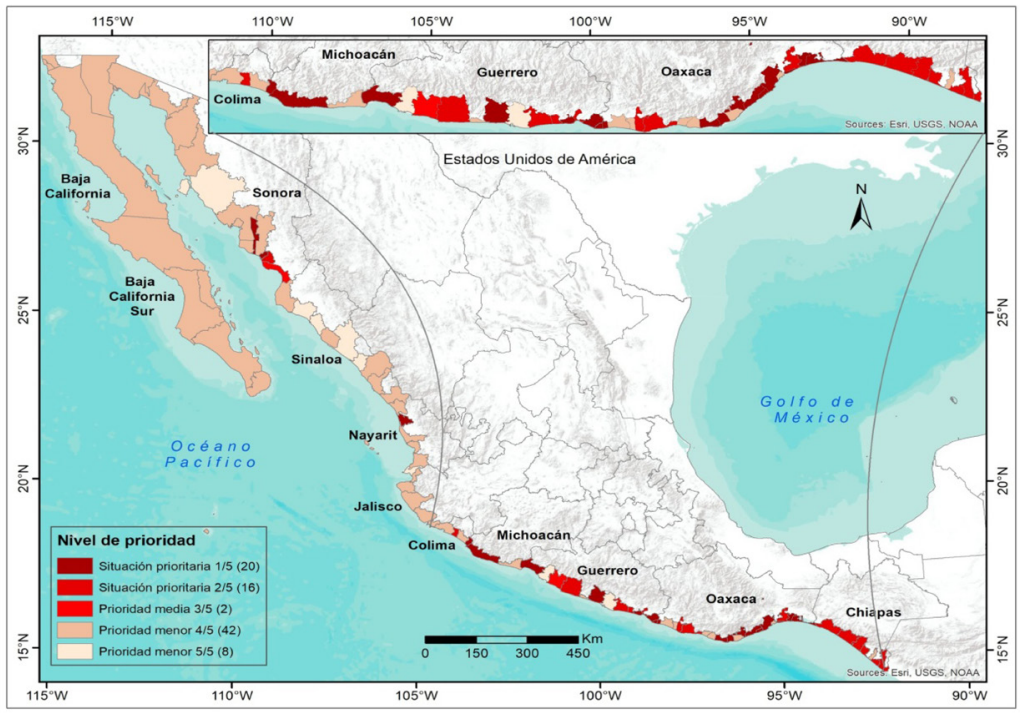

Nota: el número de municipios por nivel de prioridad se indica entre paréntesis.

Fuente: elaboración propia con base en el análisis de conglomerados e Inegi, 2010b. 


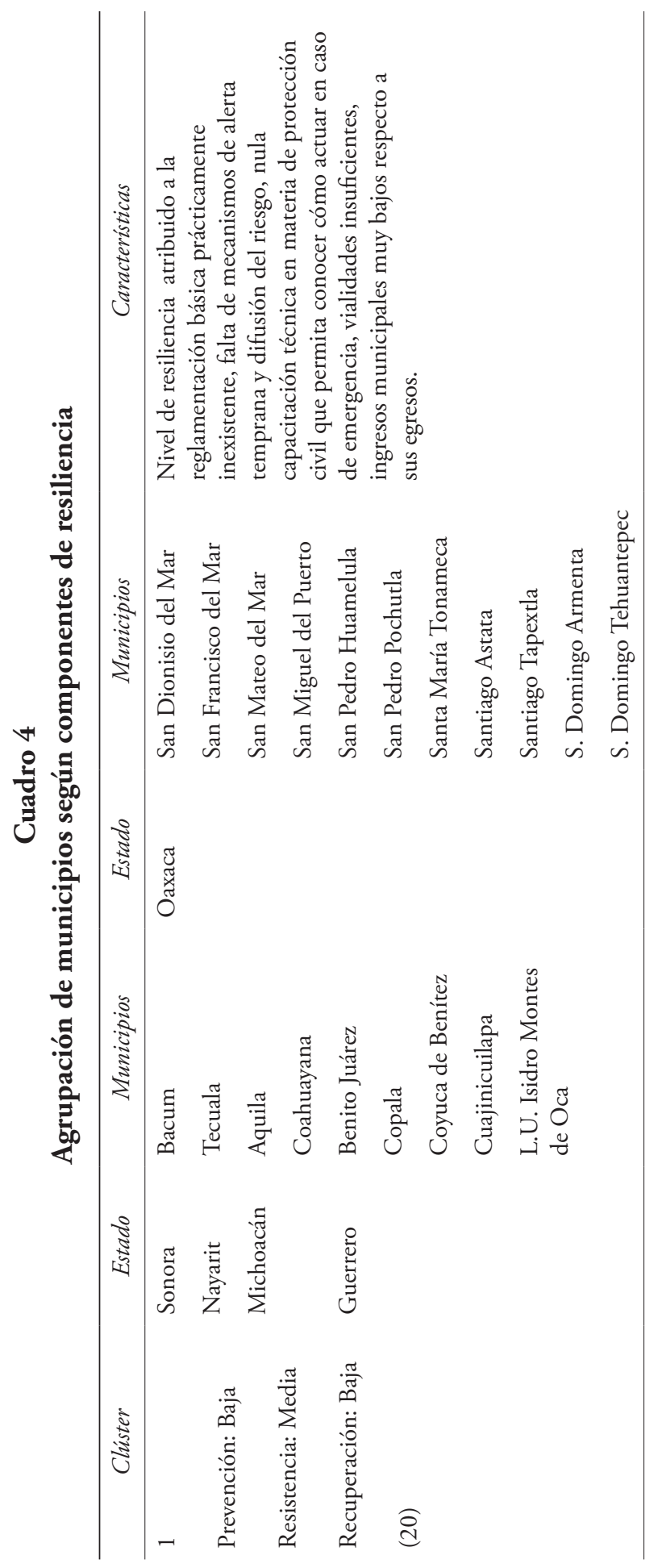




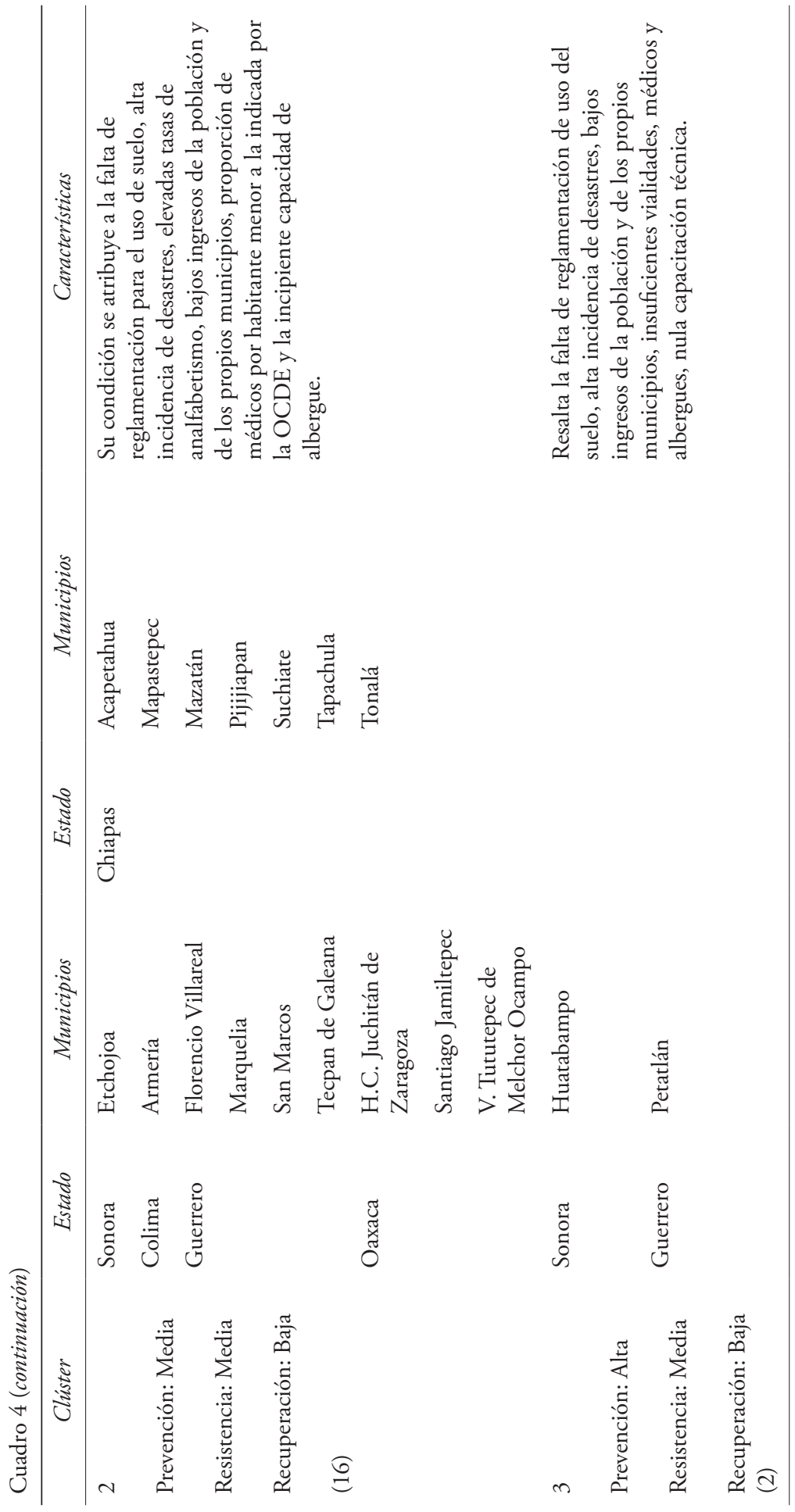




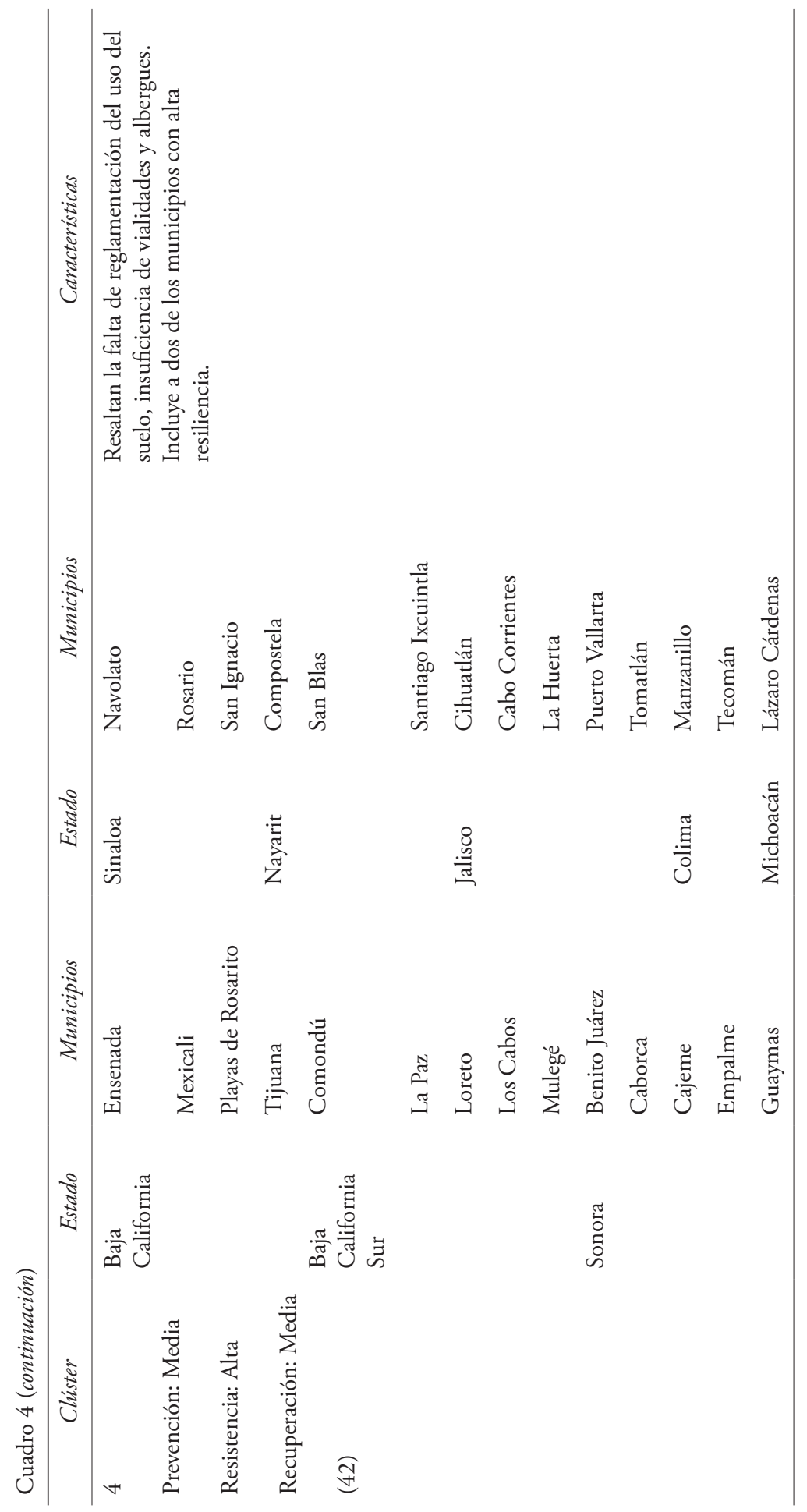




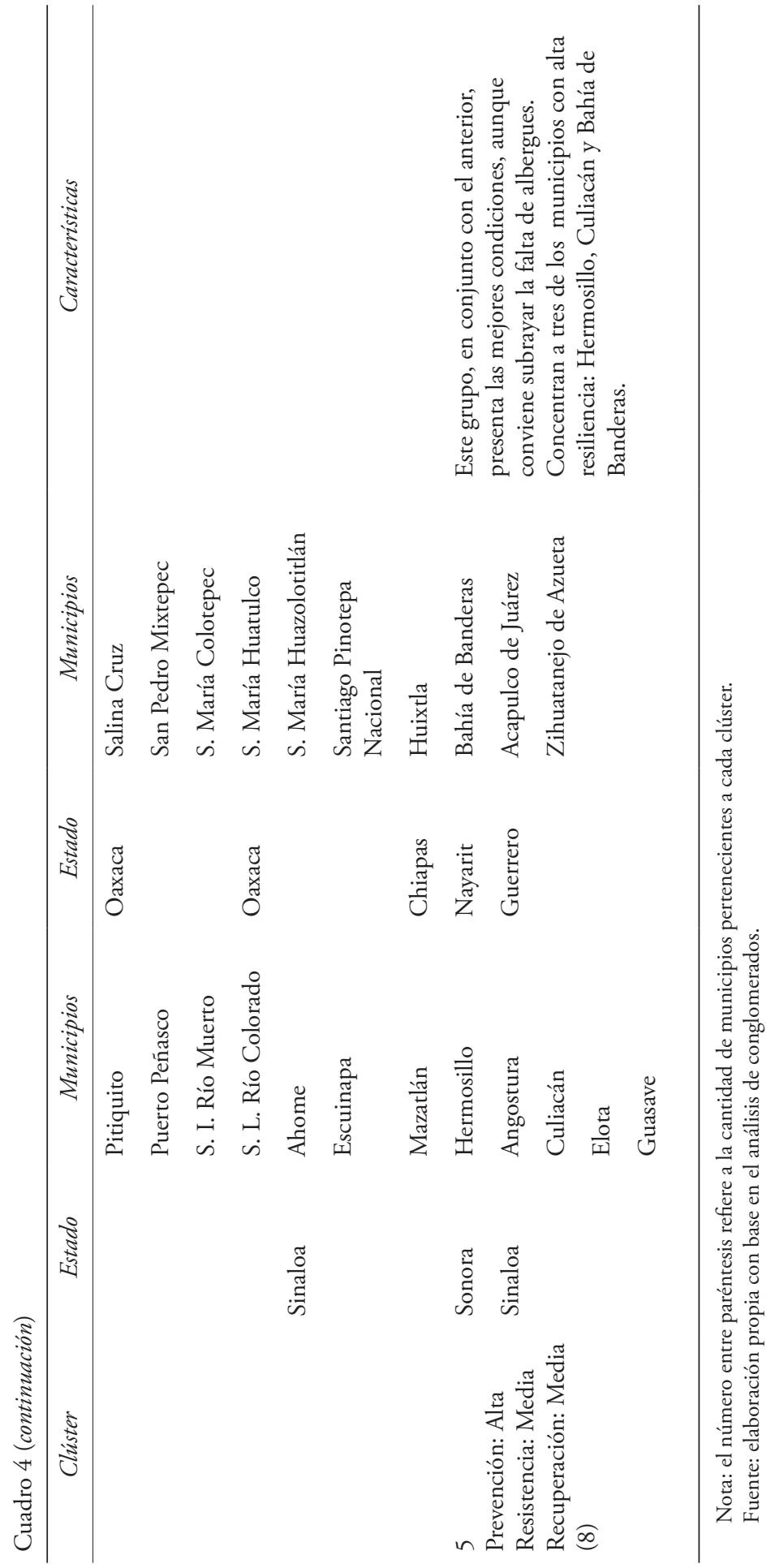


La distribución geográfica de los municipios dentro de cada clúster es heterogénea, si bien se puede distinguir cierta concentración en alguna región en particular, existen casos aislados, por lo que la atención es atribuible a diferentes niveles; por ejemplo, las acciones podrían ser a nivel regional para el clúster 4, estatales en el clúster 5. Esta heterogeneidad pudiera disminuir mediante el uso de análisis jerárquico con orientación geoestadística.

\section{Conclusiones}

El modelo desarrollado en el presente artículo circunscribe indicadores de índole ambiental, social, institucional, legal, estructural, económicos, integradores y de infraestructura, en coincidencia con la forma de abordar la resiliencia en diversos estudios existentes, no obstante, éste modelo se llevó a cabo desde una perspectiva en que la resiliencia no es entendida simplemente como la capacidad de recuperarse o regresar al estado normal antes de la ocurrencia del desastre, sino -tomando en consideración las tres etapas propias del ciclo de resiliencia que corresponden a la prevención, resistencia y recuperación- con una visión más amplia del objeto de estudio.

La manera en que se construyó el índice y los indicadores seleccionados permite su aplicación en cualquier momento para dar seguimiento y monitoreo a los cambios en la capacidad de resiliencia y, de esta manera, tener un panorama general de la efectividad de las acciones de reducción de riesgo de desastres implementadas en el municipio. Las bases de datos que dieron fundamento al estudio fueron extraídas de fuentes oficiales, de fácil acceso, algunas generadas con una periodicidad bienal o quinquenal.

La evaluación de la resiliencia muestra una tendencia hacia nivel medio, cabe destacar la ausencia de valores bajos en la zona Pacífico Norte, así como la ausencia de valores altos en la región Sur. El valor máximo alcanzado por los 88 municipios del índice de resiliencia fue solamente 2.168, lejos del nivel teórico máximo de resiliencia (3). La capacidad de recuperación resultó el punto más endeble, aun cuando el presupuesto que se destina en México a este rubro es mayor respecto a la prevención, denota así que la visión reactiva no ha sido efectiva, es necesario invertir más en la prevención si realmente se pretende cambiar la visión que predomina en materia de riesgo. En este sentido, los municipios costeros de la región Pacífico de México se encuentran en un punto intermedio de resiliencia, deben definir la arista desde la cual abordar la problemática y reorientar sus esfuerzos.

La generación de grupos, en función de las capacidades de prevención, resistencia y recuperación, permite establecer estrategias encaminadas a 
atender las debilidades particulares de cada agrupación posibilitando una mejor distribución de recursos. En este tenor, el clúster 1 -cuyos municipios se ubican en más de $50 \%$ en el estado de Oaxaca, en $25 \%$ en Guerrero, el resto distribuidos entre Michoacán, Nayarit y Sonora- es el que requiere atención inmediata, por lo que se deben enfocar los esfuerzos en estas alcaldías a fin de mejorar sus condiciones, evitando así graves daños en un futuro.

Como estudio complementario, sería conveniente incluir información cualitativa proveniente de actores clave en la gestión del riesgo que refleje particularidades del ayuntamiento, así como emplear indicadores que no fueron considerados por la poca disponibilidad de información en la dimensión espacial, al mismo tiempo, considerar la dispersión y crecimiento de la población que influyen en los indicadores de cobertura de servicios e infraestructura dentro del municipio, por otro lado, considerar su carácter urbano o rural, a nivel localidad.

Los factores que determinan la resiliencia de un municipio son diversos, no obstante, éste será resiliente siempre que exista la capacidad financiera para gestionar el riesgo, que el gobierno local y la población trabajen en conjunto y que ambas partes estén comprometidas en evitar la reconstrucción del riesgo, cuando prevalezca una articulación entre los Atlas municipales de riesgo, Ordenamientos territoriales, Planes de Desarrollo Urbano, Reglamentos de construcción y Reglamentos de zonificación, cuando se cuente con sistemas de alerta temprana, pero también cuando existan medios de comunicación eficientes y un plan de contingencias para saber cómo actuar. Finalmente, es imperante no sólo la existencia de un marco regulatorio, sino que éste se conozca, se emplee, se respete, se implemente y se actualice de manera continua.

\section{Fuentes consultadas}

Agroasemex S.A. (2017), "Base de datos (Vistas y EMISIN) de Suscripción de Reaseguro Agrícola a Fondos de Aseguramiento”, Querétaro, Agroasemex S. A. <https://datos.gob.mx/busca/dataset/bases-dedatos-vistas-y-emisin-de-suscripcion-de-reaseguro-agricola-afondos-de-aseguramiento>, 1 de marzo de 2018.

Ahmed, Iftekhar and Charlestworth, Esther (2014), "An Evaluation Framework for assessing Resilience of post-disaster Housing", International Journal of Disaster Resilience in the Built Environment, 6 (3), Melbourne, Emerald Group Publishing, pp. 300-312, doi: 10.1108/IJDRBE-11-2013-0042 
Ajibade, Idowu; McBean, Gordon and Bezner-Kerr, Rachel (2013), "Urban Flooding in Laos, Nigeria: Patterns of Vulnerability and Resilience among Women”, Global Environmental Change, 23 (6), Ámsterdam, Elsevier, pp.1714-1725.

Angeon, Valérie y Bates, Samuel (2015), "Reviewing Composite Vulnerability and Resilience Index: a Sustainable Approach and Application", World Development, vol. 72, Ámsterdam, Elsevier, pp. 140-162.

Ávila Flores, Brenda y González Gaudiano, Édgar (2015), “Sociedades resilientes: criterios para estrategias educativas encaminadas a la reducción de riesgo de desastres", Revista Interamericana de Educación de Adultos, 37 (2), Toluca, Centro de Cooperación Regional para la Educación de Adultos en América Latina y el Caribe, pp. 26-46.

Azuz-Adeath, Issac; Rivera-Arriaga, Evelia and Alonso-Peinado, Humberto (2019), "Current Demographic Conditions and Future Scenarios in Mexico's Coastal Zone”, Journal of Integrated Coastal Zone Management, 19 (2), Itajaí, Universidade do Vale do Itajaí, pp. 85-102, doi: $10.5894 /$ rcgi-n216

Baas, Stephan; Ramasamy, Selvaraju; Dey de Pryck, Jennie y Battista, Federica (2009), "Análisis de sistemas de gestión del riesgo de desastres. Una guía”, Roma, FAO.

BID (Banco Interamericano de Desarrollo) (2015), "Indicadores de riesgo de desastres y de gestión de riesgo. Programa para América Latina y el Caribe: México", Nota técnica núm. IDB-TN-800, Washington, BID, <https://publications.iadb.org/es/publications/spanish/ document/Indicadores-de-Riesgo-de-Desastre-y-deGesti\%C3\%B3n-de-Riesgos-Programa-para-Am\%C3\%A9ricaLatina-y-el-Caribe-M\%C3\%A9xico.pdf>, 1 marzo de 2020.

Briguglio, Lino; Cordina, Gordon; Farrugia, Nadia and Vella, Stephanie (2005), "Conceptualizing and Measuring Economic Resilience", en Satish Chand (eds.), Pacific Island Regional Integration and Governance, Canberra, ANU Press, pp. 265-288.

Cenapred (Centro Nacional de Prevención de Desastres) (2020), "Sistema de Consulta de Declaratorias 2000-2020”, Ciudad de México, Cenapred, <http://www.atlasnacionalderiesgos.gob.mx/apps/ Declaratorias/>, 3 agosto de 2020. 
Cenapred (Centro Nacional de Prevención de Desastres) (2015a), "Declaratorias de emergencia y desastre”, Ciudad de México, Cenapred, $<$ https://datos.gob.mx/busca/dataset/centro-nacional-de-prevencion-de-desastres/resource/fa17739f-83f2-424a-ac48 fea7c721ed 31?inner_span=True>, 1 marzo de 2018.

Cenapred (Centro Nacional de Prevención de Desastres) (2015b), "Impactos socioeconómicos de desastres de 2000 a 2015", Ciudad de México, Cenapred, <https://datos.gob.mx/busca/dataset/impacto-socioeconomico-de-desastres-de-2000-a-2015>, 1 de marzo de 2018.

Chen, Changkun; Xu, Lili; Zhao, Dongyue; Xu, Tong and Lei, Peng (2020), "A New Model for Describing the Urban Resilience Considering Adaptability, Resistance and Recovery", Safety Science, vol. 128, Ámsterdam, Elsevier, pp. 1-9, doi: https://doi. org/10.1016/j.ssci.2020.104756

Clark-Ginsberg, Aaron; McCaul, Bernard; Bremaud, Isabelle; Cáceres, Gabriela; Mpanje, Desire; Patel, Sonny and Patel, Ronak (2020), "Practitioner Approaches to Measuring Community Resilience: the Analysis of the Resilience of Communities to Disasters Toolkit", International Journal of Disaster Resilience, vol. 50, Ámsterdam, Elsevier, pp. 1-10, doi: https://doi.org/10.1016/j.ijdrr.2020.101714

Coneval (Consejo Nacional de Evaluación de la Política de Desarrollo Social) (2015), "Cohesión Social Municipios", Ciudad de México, Coneval, <https://www.coneval.org.mx/Medicion/Paginas/Cohesion_Social.aspx $>, 1$ de marzo de 2018.

Constantino, Roberto y Dávila, Hilda (2011), “Una aproximación a la vulnerabilidad y la resiliencia ante eventos hidrometeorológicos extremos en México", Politica y Cultura, núm. 36, Ciudad de México, Universidad Autónoma Metropolitana unidad Xochimilco, pp.15-44.

Contreras, Yasna del Carmen y Beltrán, Maricel (2015), "Reconstruir con capacidad de resiliencia: el casco histórico de la Ciudad de Constitución y el sitio del desastre del terremoto y tsunami del 27 de febrero 2010", Revista INVI, 30 (83), Santiago de Chile, Universidad de Chile, pp. 79-115, <http://revistainvi.uchile.cl/index. php/INVI/article/view/826/0>, 3 de agosto de 2020. 
Copeland, Samantha; Comes, Tina; Bach, Sylvia; Nagenborg, Michael; Schulte, Yannic and Doorn, Neelke (2020), "Measuring Social Resilience: Trade-offs, Challenges and Opportunities for Indicator Models in transforming Societies", International Journal of Disaster Risk Reduction, vol. 51, Ámsterdam, Elsevier, pp. 1-109, doi: https://doi.org/10.1016/j.ijdrr.2020.101799

CRED (Centre for Research on the Epidemiology of Disasters) (2018), "Natural Disaster 2018. An Opportunity to prepare, Disasters in Numbers", Bélgica, Institute Health and Society UClouvain, $<$ https://reliefweb.int/report/world/natural-disasters-2018>, 2 de marzo de 2020.

Cutter, Susan; Burton, Christopher y Emrich, Chris (2010), "Disaster Resilience Indicators for Benchmarking Baseline Conditions", Journal of Homeland Security and Emergency Management, 7 (51), Berkeley, The Berkeley Electronic Press, pp. 1-22.

Desinventar (2020), "Inventario Histórico de Desastres, Base de datos. México", Colombia, Corporación OSSO- Oficina de las Naciones Unidas para la Reducción del Riesgo de Desastres-LA RED, $<$ https://bit.ly/3gWToiU>, 01 de marzo de 2018.

Dirección General de Protección Civil (2017), "Refugios temporales zona Pacífico y Atlántico", Ciudad de México, Coordinación Nacional de Protección Civil, <https://bit.ly/2J6az5m>, 1 de marzo de 2018.

DOF (Diario Oficial de la Federación) (2015), "Reglas de operación del Programa de Prevención de Riesgos, para el ejercicio fiscal 2016", 31 de diciembre, Ciudad de México, Segob, <https://bit. ly/3nwXiBN>, 5 de marzo de 2020.

FAO (Organización de las Naciones Unidas para la Alimentación y la Agricultura) (2017), "Lineamientos y recomendaciones para la implementación del Marco de Sendai para la Reducción del Riesgo de Desastres en el Sector Agrícola y Seguridad Alimentaria y Nutricional América Latina y el Caribe”, Santiago, FAO-Oficina de las Naciones Unidas para Reducción de Riesgo de Desastres, <https://www.unisdr.org/files/50526_lineaminetos061016.pdf>, 2 de agosto de 2020. 
Fundación idea (2017), "Resiliencia urbana en América Latina: Una guía breve para autoridades locales", Ciudad de México, Fundación idea, <https://bit.ly/2KFqmIs>, 3 agosto de 2020.

GNDR (Global Network of Civil Society Organisations for Disaster Reduction) (2015), "Visión de Primera Línea 2019”, Inglaterra, GNDR, <https://www.gndr.org/es/programas/vision-de-primeralinea/vfl-2019.html>, 5 de marzo de 2020.

Goal (Organización Humanitaria Internacional) (2013), “Guía para la matriz de indicadores de resiliencia comunitaria a emergencias y desastres. Proyecto: Preparación ante desastres con enfoque comunitario, promoviendo un incremento de la coordinación en el área homogénea transfronteriza de la mosquita”, Honduras, Goal.

Gobierno Federal (2013), "Informe Nacional del Progreso en la Implementación del Marco de Acción de Hyogo (2011-2013)", Ciudad de México, Sistema Nacional de Protección Civil (Sinaproc), $<$ https://bit.ly/3r8D57C >, 5 de marzo de 2020.

Gobierno Federal (2008), "Primer Informe de México sobre la aplicación y seguimiento del Marco de Acción de Hyogo 2005-2010", Ginebra, Plataforma Global para la Reducción del Riesgo de Desastres, <https://www.preventionweb.net/files/7977_PrimerInformedeSP.pdf>, 5 de marzo de 2020.

Gobierno Federal (s.f.), “Ámbito municipal, compilación jurídica”, Ciudad de México, Unidad General de Asuntos Jurídicos, <http:// www.ordenjuridico.gob.mx/ambmun.php\#gsc.tab=0>, 1 de marzo de 2018.

González, Manuel Ramón y Franco, Nadia (2017), "Eventos naturales críticos y su impacto en el turismo: reflexiones desde la sustentabilidad en la Ciudad Juárez, México", Academia Journal, 9 (2), San Antonio Texas, Publicaciones del Congreso Internacional de Investigación Academia Journals, pp. 410-415.

Inafed (Instituto Nacional para el Federalismo y el Desarrollo Municipal) (2016a), "Índice de reglamentación municipal básica 2008-2014", Ciudad de México, Inafed, <http://www.inafed.gob.mx/es/inafed/ Desempeno_Institucional_Municipal>, 1 de marzo de 2018. 
Inafed (Instituto Nacional para el Federalismo y el Desarrollo Municipal) (2016b), "Finanzas Públicas", Ciudad de México, Inafed, <http:// www.inafed.gob.mx/es/inafed/Desempeno_Institucional_Municipal>, 1 de marzo de 2018.

Inafed (Instituto Nacional para el Federalismo y el Desarrollo Municipal) (2015), "Policías por cada 100 mil habitantes, 2008-2012", Ciudad de México, Inafed, <http://www.inafed.gob.mx/es/inafed/ Desempeno_Institucional_Municipal>, 1 de marzo de 2018.

Inegi (Instituto Nacional de Estadística y Geografía) (2017), “Anuario estadístico y geográfico por entidad federativa 2016”, Ciudad de México, Inegi, <https://www.inegi.org.mx/app/biblioteca/ficha. html?upc=702825107017>, 1 de marzo de 2018.

Inegi (Instituto Nacional de Estadística y Geografía) (2015), "Censo Nacional de Gobiernos Municipales y Delegacionales 2015", Ciudad de México, Inegi, <https://www.inegi.org.mx/programas/ cngmd/2015/default.html\#Microdatos>, 1 de marzo de 2018.

Inegi (Instituto Nacional de Estadística y Geografía) (2013), "Uso de suelo y vegetación, serie V", Ciudad de México, Inegi, <www. inegi.org.mx/temas/usosuelo/default.html\#Descargas $>$, $1 \mathrm{de}$ marzo de 2018.

Inegi (Instituto Nacional de Estadística y Geografía) (2010a), “Censo de Población y Vivienda 2010”, Ciudad de México, Inegi, <https:// www.inegi.org.mx/programas/ccpv/2010/>, 1 de marzo de 2018.

Inegi (Instituto Nacional de Estadística y Geografía) (2010b), "Marco Geoestadístico 2010”, Ciudad de México, Inegi, <https://www. inegi.org.mx/programas/ccpv/2010/>, 1 de marzo de 2018.

IBM (International Business Machines) (2009), "Statistical Package for the Social Sciences”, version 23, New York, IBM.

Jones, Keith; Brydson, Helen; Ali, Faud and Cooper, Justine (2013), "Assessing Vulnerability, Resilience and Adaptive Capacity of a UK Social Landlord", International Journal of Disaster Resilience in the Built Environment, 4 (3), Melbourne, Emerald Group Publishing, pp. 287-296, doi: 10.1108/IJDRBE-03-2013-0004 
Labaka, Leire; Hernantes, Josune and Sarriegi, Jose Mari (2015), "A Framework to Improve the Resilience of Critical Infrastructures", International Journal of Disaster Resilience in the Built Environment, 6 (4), Melbourne, Emerald Group Publishing, pp. 409-423, doi: 10.1108/IJDRBE-07-2014-0048

Lu, Xinzheng; Liao, Wenjie; Fang, Dongping; Lin, Kaiqi; Tian, Yuan; Zhang, Chi; Zheng, Zhe and Zhao, Pengju (2020), "Quantification of Disaster Resilience in Civil Engineering: a Review", Journal of Safety Science and Resilience, 1 (1), Ámsterdam, Elsevier, pp. 19-30, doi: https://doi.org/10.1016/j.jnlssr.2020.06.008

Maldonado, Ana Lucía y González, Édgar (2013), “De la resiliencia comunitaria a la ciudadanía ambiental. El caso de tres localidades en Veracruz, México", Integra educativa, 6 (3), La Paz, Instituto Internacional de Integración, pp. 13-28, <http://www.scielo.org. bo/pdf/rieiii/v6n3/n6a02.pdf>, 5 de marzo de 2020.

Medina, María de la Paz; Olguín, Mónica Paola; Solorio, Alma Rosa y Sansón, Leodegario (2017), "Estudio estratégico. Inventario de Atlas de Riesgos en México. Informe del estado actual", Ciudad de México, Academia de Ingeniería México, <https://www.ai.org. $\mathrm{mx} /$ sites/default/files/atlas_riesgo.pdf>, 5 de marzo de 2020.

Metzger, Pascale y Robert, Jérémy (2013), “Elementos de reflexión sobre la resiliencia urbana: usos criticables y aportes potenciales", Territorios, núm. 28, Bogotá, Universidad del Rosario, pp. 21-40.

Montalba, René; García, Marcia; Altieri, Miguel; Fonseca, Francisca y Vieli, Lorena (2013), "Utilización del índice holístico de riesgo (IHR) como media de resiliencia socioecológica a condiciones de escasez de recursos hídricos. Aplicación en comunidades campesinas e indígenas de la Araucanía, Chile”, Agroecología, 8 (1), Murcia, Universidad de Murcia, pp. 63-70.

OCDE (Organización para la Cooperación y el Desarrollo Económico) (2013), "Estudio de la OCDE sobre el Sistema Nacional de Protección Civil en México”, Ciudad de México, OCDE.

Patel, Sonny; Rogers, Brooke; Amlôt, Richard; Rubin, James (2017), "What do we mean by 'Community Resilience'? A Systematic Literature Review of how it is defined in the Literature", PloS 
Currents, vol. 9, San Francisco, Public Library of Science, doi: 10.1371/currents.dis.db775aff25efc5ac4f0660ad9c9f7db2

Rosengaus, Michel; Jiménez, Martín y Vázquez, María Teresa (2014), "Atlas climatológico de ciclones tropicales en México", Ciudad de México, Centro Nacional de Prevención de Desastres-Instituto Mexicano de Tecnología del Agua, <http://www.cenapred.gob. $\mathrm{mx} / \mathrm{es} /$ Publicaciones/archivos/37.pdf $>, 5$ de marzo de 2020.

Santiago Vera, Teresita de Jesús; García Millán, Máximo y Rosset, Peter (2018), "Enfoques de la resiliencia ante el cambio climático", Agricultura, Sociedad y Desarrollo, 15 (4), Texcoco, Colegio de Postgraduados, pp. 531-539.

Schuschny, Andrés y Soto, Humberto (2009), "Guía metodológica. Diseño de indicadores compuestos de desarrollo sustentable", Santiago de Chile, Comisión Económica para América Latina y el Caribe.

Sedano, Ruth (2012), "Gestión integrada del riesgo de inundaciones en Colombia”, tesis de maestría, Universidad Politécnica de Valencia, Valencia.

Sedatu (Secretaría de Desarrollo Agrario, Territorial y Urbano) (s.f.), "Cobertura de Atlas Municipales", Ciudad de México, Sedatu, $<$ http://www.atlasnacionalderiesgos.gob.mx/archivo/cob-atlasmunicipales.html>, 1 de marzo de 2018.

SGM (Servicio Geológico Mexicano) (2017), "Sismología de México. Museo virtual”, Ciudad de México, SGM, <http://www.sgm.gob. $\mathrm{mx} / \mathrm{Web} /$ MuseoVirtual/Riesgos-geologicos/Sismologia-deMexico.html>, 5 de marzo de 2020.

SMIE (Sociedad Mexicana de Ingeniería Estructural, A.C.) (s.f.), "Reglamentos de Construcción México", Ciudad de México, SMIE, $<$ https://bit.ly/37vJvpp>, 1 de marzo de 2018.

Tumini, Irina (2016), "Acercamiento teórico para la integración de los conceptos de Resiliencia en los indicadores de Sostenibilidad Urbana”, Revista de Urbanismo, núm. 34, Santiago de Chile, Universidad de Chile, pp. 4-19, doi:10.5354/07175051.2016.40056 
Twigg, John (2007), "Características de una comunidad resiliente ante los desastres. Nota guía”, Suiza, Oficina de Naciones Unidas para la Reducción del Riesgo de Desastres, <https://bit.ly/34n23X6>, 1 de agosto de 2019.

Uriarte Arciniega, Juan de Dios (2013), "La perspectiva comunitaria de la resiliencia”, Psicología política, núm. 47, Valencia, Universidad de Valencia, pp. 7-18.

Recibido: 12 de mayo de 2020.

Reenviado: 4 de agosto de 2020. Aceptado: 17 de septiembre de 2020.

Karen Velázquez-González. Maestra en Ciencias por la Universidad Autónoma del Estado de México. Actualmente es profesora de asignatura en la Facultad de Ciencias Marinas de la Universidad Autónoma de Baja California. Sus líneas de investigación actuales son: resiliencia, riesgo y ordenamiento territorial. Ha participado en la elaboración de Atlas Municipales de Riesgos Naturales y Programas de Ordenamiento Ecológico. Entre sus más recientes publicaciones se encuentran, en coautoría: "Respuesta al comentario por Juan José Muñoz Pérez y Gregorio Gómez Pina sobre: Variaciones sedimentarias y transporte litoral en Playa de la Victoria, Cádiz, España”, Revista Ciencias Marinas, núm. 1, Ensenada, Baja California, Instituto de Investigaciones Oceanológicas, pp. 105-106 (2010); "Variaciones sedimentarias y transporte litoral en Playa de la Victoria, Cádiz, España", Revista Ciencias Marinas, 36 (1), Ensenada, Baja California, Instituto de Investigaciones Oceanológicas, pp. 259-269 (2009).

Salvador Adame-Martínez. Doctor en Ciencias por el Colegio de Postgraduados. Actualmente es profesor-investigador en el Centro de Investigación y Estudios Avanzados en Planeación Territorial, Facultad de Planeación Urbana y Regional de la Universidad Autónoma del Estado de México. Es miembro del Sistema Nacional de Investigadores, nivel I. Líneas de investigación actuales: riesgo y vulnerabilidad, variabilidad climática, cambios de uso de suelo, sustentabilidad urbana. Entre sus más recientes publicaciones destacan, en coautoría: "Landslides in Mexico: their Occurrence and Social Impact since 1935", Landslides, núm. 17, Berlín, Springer, pp. 379-394 (2020); "Sustentabilidad del desarrollo urbano en la Megalópolis de México: perspectiva desde el análisis espacial”, Economía, Sociedad y Territorio, 20 (62), Zinacantepec, El Colegio Mexiquense 
A. C., pp. 89-122 (2020); "Análisis de la distribución espacial de los RSU en la Zona Metropolitana de Tampico", Revista Quivera, núm. 2, Toluca, Universidad Autónoma del Estado de México, pp. 83-111 (2019).

Guadalupe Hoyos Castillo. Maestra en Desarrollo Municipal, con estudios concluidos en Doctorado en Urbanismo. Actualmente está adscrita a la Facultad de Planeación Urbana y Regional de la Universidad Autónoma del Estado de México. Líneas de investigación: procesos de urbanización y metropolización, interacciones metropolitanas suelo y agua y, economía urbana. Entre sus más recientes publicaciones destacan, en coautoría: "Reajustes asimétricos en la relación rural-urbana. Poniente del Estado de México", en Juan Roberto Calderón Maya y Carlos Alberto Pérez Ramírez (coord.), Ciudad y ambiente: procesos y transformaciones en contextos urbanos (próximamente); "Sistema urbano y rural desde lo regional en México”, en José Gasca (coord.), Desarrollo regional en México. Actores, miradas y relatos, Ciudad de México, UNAM-AMECIDER, pp. 247-268 (2019); "Desigualdad socio-espacial, innovación tecnológica y procesos urbanos", en José Gasca y Eréndira Serrano (coord.), Regiones, desplazamientos y geopolítica. Agenda pública para el desarrollo territorial, vol. 3, Ciudad de México, UNAM-AMECIDER, (2019); "Estructuración espacial de las relaciones funcionales metropolitanas: el caso de Toluca, 2000-2015", en Guadalupe Hoyos, Serena Serrano y María del Pilar Mora (coord.), Ciudad, género, cultura y educación en las regiones, Ciudad de México, UNAM/IIEc-AMECIDER, pp. 301-330 (2017).

Georges Seingier. Doctor en Medio Ambiente y Desarrollo por la Universidad Autónoma de Baja California. Actualmente es profesor-investigador en la Facultad de Ciencias Marinas de la Universidad Autónoma de Baja California. Líneas de investigación: prospectiva, análisis espacial y sus aplicaciones en la gestión sustentable del espacio costero. Entre sus más recientes publicaciones destacan, en coautoría: "Gobernanza ambiental ante el Cambio Climático: municipios costeros de México”, en Rivera-Arriaga, Evelia; Azuz-Adeath, Isaac; Cervantes Rosas, Omar; Espinoza-Tenorio, Alejandro; Silva Casarín, Rodolfo; Ortega-Rubio, Alfredo; Botello, Alfonso y Vega Serratos, Beatriz (eds.), Gobernanza y manejo de las costas y mares ante la incertidumbre. Una guia para tomadores de decisiones, Campeche, Universidad Autónoma de Campeche, pp. 93-116 (2020); "Status and scope of marine mammal stranding research in Mexico", Journal of Coastal Conservation, 24 (3), Berlín, Springer, pp. 396-411 (2020); "Indicators to Measure Pressure, State, Impact and Responses of Surf Breaks: the Case Of Bahía De Todos Santos World Surfing Reserve", Ocean and Coastal Management, vol. 194, article 105252, Ámsterdam, Elsevier; pp. 1-11 (2020). 